\title{
Obtención de aceite esencial de romero con fines cosméticos
}

Vélez Mendoza Biuty Sherina1D; Pita Meza María Nicole ${ }^{1}$; Moreira Mendoza Karen Estefania1 ${ }^{1}$; Pin Mera Diana Elizabeth ${ }^{1}$; Oyervide Vargas Ariane Monserrat 1 ; María Antonieta Riera ${ }^{2} \odot$

${ }^{1}$ Estudiantes de la Facultad de Ciencias Matemáticas, Físicas y Químicas, Carrera de Ingeniería Química, Universidad Técnica de Manabí., 2Facultad de Ciencias Matemáticas, Físicas y Químicas, Universidad Técnica de Manabí. 1bvelez7151@utm.edu.ec; 1mpita8965@utm.edu.ec; 1kmoreira4312@utm.edu.ec; 1dpin4761@utm.edu.ec; 1aoyervide4815@utm.edu.ec; 2mriera@utm.edu.ec DOI https://doi.org/10.33412/pri.v10.1.2170

\section{(1)(9)}

Resumen: Los aceites esenciales son extractos que pueden ser utilizados en distintos sectores de la industria. El presente trabajo de investigación se realizó en la asignatura Cálculo a la Ingeniería Química I, con el objetivo de desarrollar un proceso a nivel experimental donde fuese posible realizar el balance de masa y que además sirviera de referencia a nivel industrial. El mismo se dividió en dos etapas: primero, la extracción del aceite esencial de romero por el método destilación por arrastre de vapor donde se obtuvo un rendimiento de 0,53\%; segundo, se realizó una saponificación donde se utilizó el aceite de romero como un aromatizante, obteniendo finalmente un jabón de tocador de $\mathrm{pH}$ básico. El procedimiento realizado permitió comprobar que es posible utilizar aceites esenciales para un fin diferente al comúnmente empleado, sirviendo así como referencia para industrias de cosméticos con interés en desarrollar productos basados en extractos naturales.

Palabras clave: Aceite esencial, romero, destilación por arrastre de vapor, jabón de tocador.

Title: Obtaining rosemary essential oil for cosmetic purposes.

Abstract: Essential oils are extracts that can be used in different sectors of the industry. The present research work was carried out in the matter Calculus to Chemical Engineering I, with the objective of developing a process at experimental level where the mass balance could be made and that will serve a reference at an industrial level. It was divided into two stages: First the extraction of rosemary essential oil by the steam distillation method where a yield of $0.53 \%$ was obtained. Later, a saponification was carried out where the rosemary oil was used as a fragance agent, finally obtaining a basic $\mathrm{pH}$ toilet soap.

The procedure carried out proved that it is possible to use essential oils for a purpose other than that commonly used, thus serving as a reference for cosmetic industries interested in developing products based on natural extracts.

Key words: Essential oil, rosemary, steam distillation, toilet soap.

Tipo de artículo: original

Fecha de recepción: 20 de agosto de 2018

Fecha de aceptación: 17 de octubre de 2018

\section{Introducción}

El romero (Rosmarinus officinalis L.) es una planta aromática conocida y utilizada desde la antigüedad como condimento y con fines medicinales. Puede medir entre 50 y $150 \mathrm{~cm}$ de altura y sus hojas hasta $3 \mathrm{~cm}$ de largo y $4 \mathrm{~mm}$ de ancho. Las hojas de romero es el lugar donde se concentran los principios activos de la planta y poseen entre 1,0 y $2,5 \%$ de aceite esencial, además de diterpenos, flavonoides y polifenoles [1].

Los aceites esenciales (AEs) son líquidos aromáticos y volátiles obtenidos de las plantas, incluyendo flores, raíces, corteza, hojas, semillas, cáscara, frutas, madera y plantas enteras [2]. Estos contienen entre 85 y $99 \%$ de componentes volátiles y entre 1 y $15 \%$ de componentes no volátiles [3].

El aceite esencial es el compuesto más estudiado de la planta debido a que los principios bioactivos que lo conforman, tienen importantes propiedades biológicas como lo son su actividad antioxidante, citotóxica y anticancerígena [4].

Este se caracteriza por ser un líquido transparente y muy fluido, con un olor y sabor penetrante, aromático y alcanforado. Su composición aproximada es $21,9 \%$ piperitona, $14,9 \%$ apineno, $14,9 \%$ linalool [5], además de otros entre los que se encuentra el 1,8-cineol, alcanfor, alfa-terpineol, canfeno, borneol, acetato de bornilo, limoneno, mirceno, verbenona. Sin embargo, esta puede variar dependiendo de la parte de la planta recolectada, el grado de desarrollo en el momento de su recolección, la procedencia geográfica, entre otros [1].

Existen varios métodos para extraer aceites esenciales. Estos pueden incluir el uso de dióxido de carbono líquido o microondas, aunque comúnmente se usa destilación a baja 0 alta presión empleando agua hirviendo o vapor de agua [6].

La destilación por arrastre consiste en vaporizar dos líquidos inmiscibles a una temperatura menor a la de ebullición de cada uno de los componentes volátiles presentes, usando para ello una corriente directa de vapor agua. El vapor calienta la mezcla hasta su punto de ebullición y luego los vapores generados se condensan y finalmente los dos líquidos inmiscibles (agua y aceite esencial), se separan por gravedad [7].

Este método permite la máxima difusión del vapor a través del material vegetal, reduciendo los daños que pudieran sufrir los componentes de las esencias extraídas [8]. El rendimiento obtenido en la extracción en general es muy variado entre $0,01 \%$ y $2 \%$ y varía en función de la composición de la planta así como del método utilizado [9].

Los aceites esenciales se emplean como principios activos 0 excipientes de medicamentos y en la industria agroalimentaria 
para producir bebidas y aromatizar alimentos. También tienen aplicación en la fabricación de perfumes, cosméticos, jabón, champús o geles limpiadores. Desde el punto de vista económico, es de gran interés en la industria de cosméticos, jabones, detergentes y perfumes [10].

En la actualidad ha crecido el interés en el consumo de productos cosméticos naturales formulados con ingredientes funcionales y de origen botánico. El aceite esencial juega un rol importante no sólo como fragancia, sino también como preservante del producto al prevenir la pérdida de ingredientes aromáticos volátiles [11].

Kareru et. al. [12] usaron extractos de las plantas Tithonia diversifolia Helms, Azadirachta indica y Aloe secundiflora para elaborar jabones, teniendo como resultado un producto antimicrobiano, debido a los efectos inhibidores que poseen los fitoconstituyentes de las plantas.

Un jabón contiene sales de sodio o potasio de los ácidos grasos, producto de la mezcla de un cuerpo graso (triglicéridos con un álcali, que puede ser hidróxido de sodio o de potasio) y que se obtiene a partir de una reacción llamada saponificación [13].

Los AEs pueden emplearse como fragancias naturales en la elaboración de jabón. A nivel comercial es común encontrar jabones de tocador elaborados a base de extractos de manzanilla, caléndula, ginseng, jazmín, rosa mosqueta, lavanda, eucalipto, sándalo, sábila, canela, entre otros.

El romero, la salvia, la camomila, el lúpulo y la limonaria, son algunas hierbas beneficiosas usadas para el realce visual y aromático del jabón, así como para conferir propiedades suavizantes e hidratantes en la piel [14].

Expertos aseguran que el aceite esencial de romero tiene un gran potencial para ser usado en aplicaciones farmacológicas y biotecnológicas. Pero también puede ser explotado en diferentes productos comerciales como suplemento, cosméticos y nuevas formulaciones [15].

Partiendo de estas aseveraciones se realizó el presente estudio con el objetivo de evaluar el uso del aceite esencial de romero en la elaboración de un producto cosmético, como una alternativa de uso en esta industria. Para ello se extrajo el aceite esencial de las hojas de romero y luego se utilizó en la elaboración de un jabón de tocador.

Este trabajo establece el procedimiento a seguir en la obtención de este producto cosmético y parte de una escala de laboratorio que posteriormente puede ser escalable al sector manufacturero.

\section{Materiales y métodos}

Para dar cumplimiento al objetivo se llevaron a cabo dos pasos. El primero de ellos fue la extracción del aceite esencial de romero y luego se procedió a elaborar el jabón de tocador.

\subsection{Extracción del aceite esencial}

Se seleccionaron las hojas de romero a utilizar, eliminando las hojas extrañas, tallos gruesos y tierra que pudiera estar adherida. El procedimiento para la extracción por arrastre de vapor fue el descrito por Castillo y Mendoza [16].

Se pesó una muestra de $100 \mathrm{~g}$ de hojas de romero en una balanza analítica ADAM y se redujo su tamaño para aumentar la superficie de contacto. Se colocaron en un balón de tres bocas de $1000 \mathrm{~mL}$ que estaba conectado al equipo de destilación por arrastre de vapor, conformado a su vez por un balón generador de vapor de $1000 \mathrm{~mL}$, un matraz de $250 \mathrm{~mL}$, dos placas calefactoras digitales IKA C-MAG HS7, un termómetro de vidrio, un condensador, conexiones de vidrio y goma (figura 1).

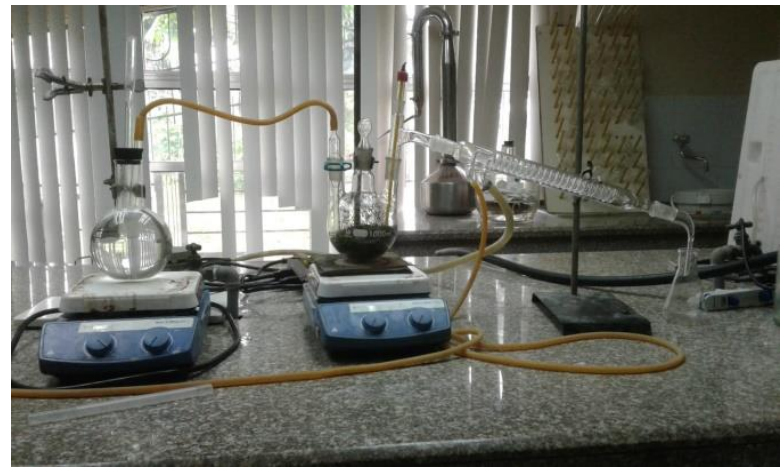

Figura 1. Equipo de destilación por arrastre de vapor.

Se calentó el balón donde se encontraba el agua hasta su ebullición, con el fin de generar el vapor y pasarlo al segundo balón para extraer la esencia de romero. Conforme se efectuó la destilación se recibió en un matraz una mezcla de aceite y agua. Se suspendió el calentamiento cuando se había consumido entre el $70 \%$ y $85 \%$ del agua alimentada.

La mezcla de agua y aceite obtenida se colocó en un embudo de separación para separar por diferencia de densidades el aceite esencial de romero del agua (figura 2). Se determinó con ayuda de una pipeta la cantidad de aceite extraído y luego se almacenó en un tubo de ensayo.

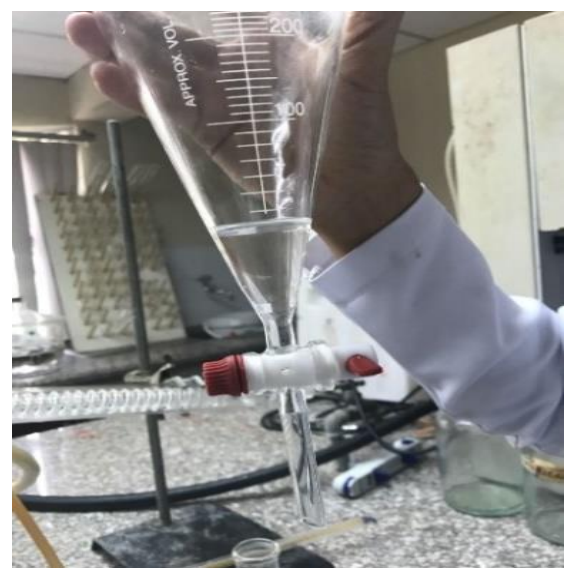

Figura 2. Equipo de destilación por arrastre de vapor. 
Para determinar la humedad tanto de las hojas de romero en la alimentación como del aceite esencial extraído, se utilizó una termobalanza MB45 OHAUS.

El porcentaje de rendimiento de aceite esencial (\%AEs), se determinó usando el método gravimétrico - volumétrico [17], a través de la ecuación (1):

$$
\% A E s=\frac{\mathrm{mAEs}(\mathrm{g})}{\mathrm{mCV}(\mathrm{g})} \times 100 \%
$$

Donde mAEs es la masa del aceite esencial obtenido en la extracción y mCV es la masa de la carga vegetal de la muestra a destilar.

\subsection{Fabricación de jabón de tocador}

Para la obtención del jabón de tocador se siguió la metodología descrita en un trabajo realizado para el diseño de una planta de fabricación de jabón a partir de aceites vegetales usados [18].

Se comenzó preparando una infusión con $200 \mathrm{~mL}$ de agua y $10 \mathrm{~g}$ de romero seco. Se calentó hasta su ebullición en una placa de calentamiento y posteriormente se retiró el romero de tal manera que solo quedara la solución acuosa (figura 3).

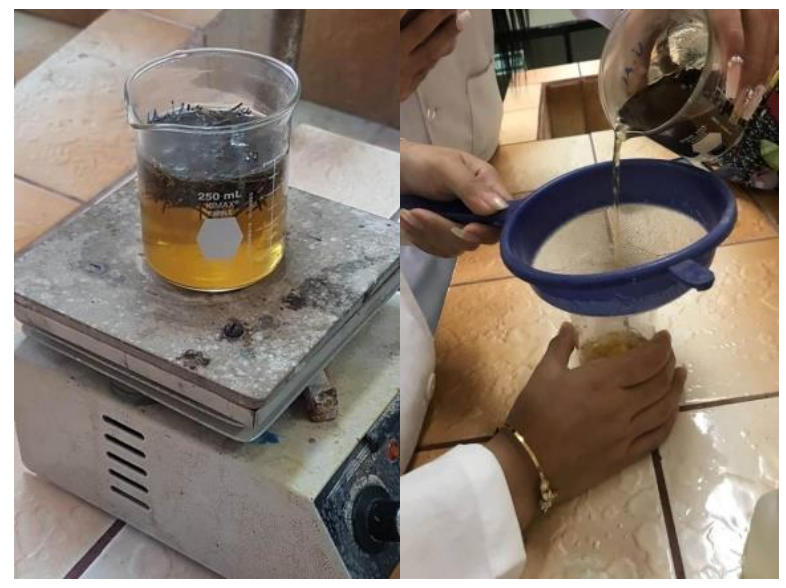

Figura 3. Preparación de la infusión de romero

Se pesaron $13,11 \mathrm{~g}$ de $\mathrm{NaOH}$ grado analítico en una balanza analítica SARTORIUS y se mezcló con 42,28 mL de la infusión preparada. Paralelamente se pesaron $100 \mathrm{gr}$ de aceite de palma comercial y se procedió a calentar por 10 minutos hasta alcanzar una temperatura de $40^{\circ} \mathrm{C}$

Transcurrido el tiempo señalado, se añadió el aceite de palma a la mezcla anteriormente preparada y se procedió a homogenizar. En este momento se lleva a cabo la reacción de saponificación (2).

$\mathrm{C}_{57} \mathrm{H}_{104} \mathrm{O}_{6}+3 \mathrm{NaOH} \rightarrow 3 \mathrm{C}_{18} \mathrm{H}_{33} \mathrm{O}_{2} \mathrm{Na}+\mathrm{C}_{3} \mathrm{H}_{5}(\mathrm{OH})_{3}$

Una vez que la mezcla espesó, se añadió el aceite esencial extraída y romero seco. Se procedió a tomar el pH con tirillas de $\mathrm{pH}$ y la mezcla obtenida se dejó reposar en un molde durante 24 horas, obteniendo finalmente el jabón de tocador.

Con los datos obtenidos durante cada proceso, se realizó un balance de masa sin reacción química (extracción de aceite esencial) y con reacción química (elaboración de jabón) en régimen estacionario [19] usando la ecuación para tal fin (2):

Entrada + Generación - Salida - Consumo = Acumulación (2)

\section{Resultados y discusión}

El proceso de extracción por el método de destilación por arrastre de vapor quedó conformado por cuatro operaciones unitarias: molienda, mezclado, destilación. El mismo se representó gráficamente a partir de un diagrama de bloques (figura 4), donde se muestran los resultados obtenidos experimentalmente además de los calculados a través de balance de masa.

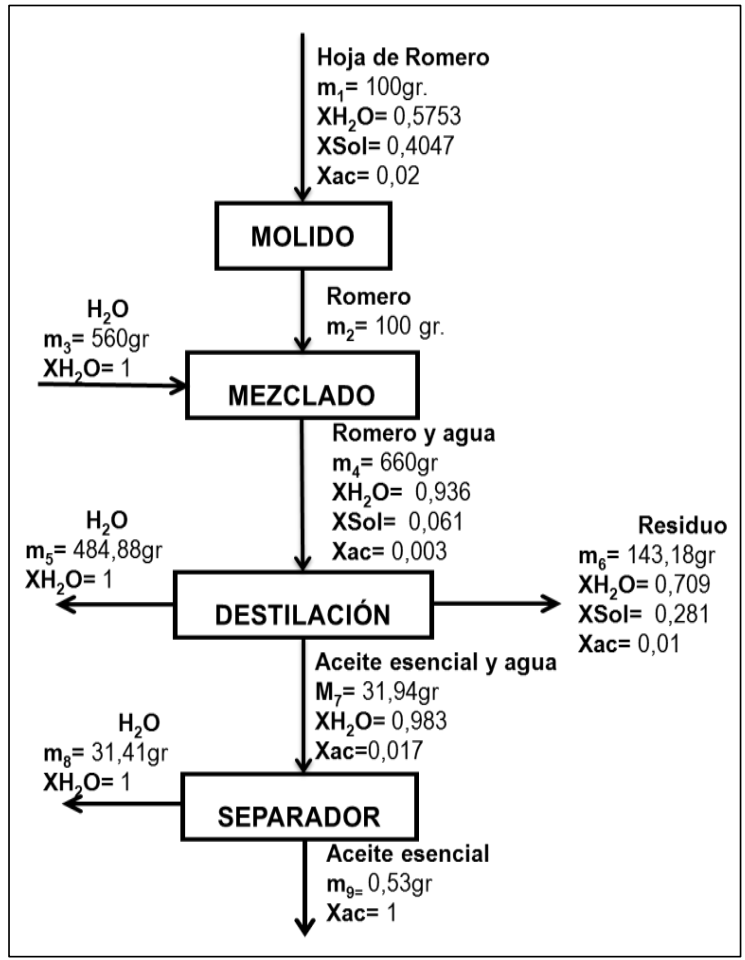

Figura 4. Diagrama de bloques para la extracción de aceite esencial de romero

A partir de $100 \mathrm{~g}$ de materia vegetal se obtuvo $0,53 \mathrm{~g}$ de aceite esencial o bien $0,58 \mathrm{~mL}$ determinada con su densidad la cual es de 0,9116 $\mathrm{g} / \mathrm{mL}$ [20]. Usando (1) se determinó el rendimiento del aceite esencial en el proceso de extracción, siendo de 0,53\%.

El resultado obtenido se encuentra dentro del rango de 0,48 y $1,75 \%$ reportado por Angioni et. al. [21] al usar este método de destilación por arrastre de vapor. Valores similares reportan Bonilla et. al. [22] con $0,67 \%$ al utilizar este mismo método de extracción, además de Valverde y Leonardo [23], quienes obtuvieron $0,61 \%$ en la extracción de aceite con tejido en estado fresco y aumentó a 0,98\% con tejido seco. Mejores rendimientos se podrían obtener si las hojas de romero se someten a secado antes de la extracción. 
El proceso de obtención de jabón se constituyó de tres operaciones unitarias: mezclado, reacción y separación. Este también se representó gráficamente con un diagrama de bloques (figura 5), señalando las operaciones unitarias realizadas para tal fin.

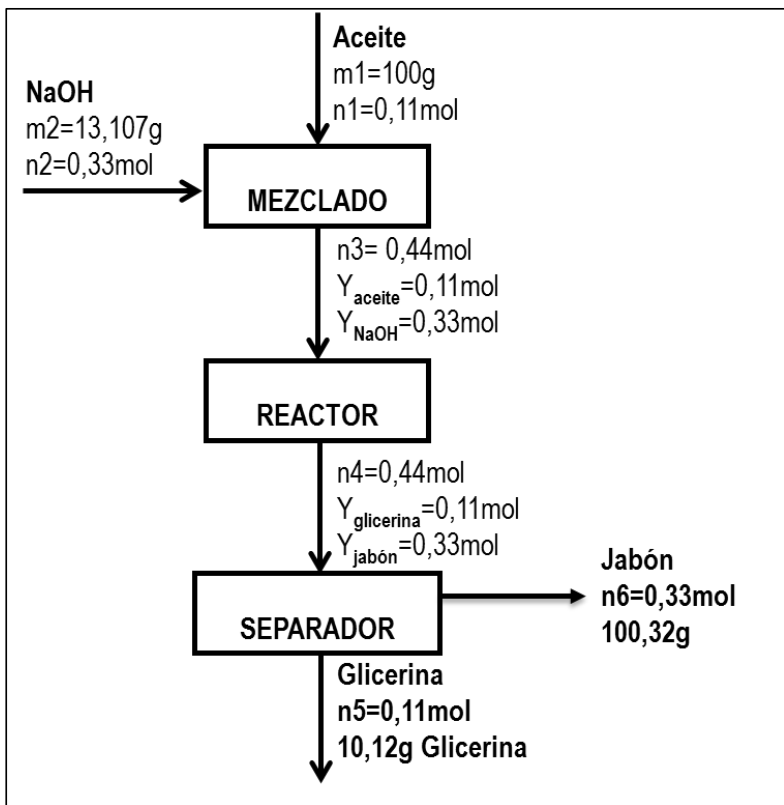

Figura 5. Diagrama de bloques para la elaboración de jabón

Al llevar a cabo la reacción de saponificación se obtuvo un jabón de tocador de 100,32 g (figura 6). Debido a que el rendimiento en la reacción calculado por estequiometria supera el $100 \%$, indica que en el jabón elaborado quedaron restos de glicerina que no se lograron separar.

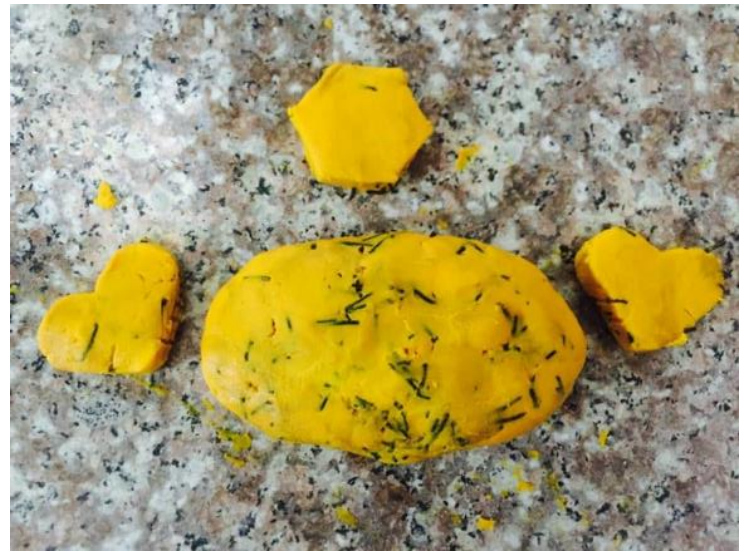

Figura 6. Jabón de tocador obtenido

El pH del jabón determinado con tirillas de $\mathrm{pH}$ fue de 8 , estando dentro del rango de $\mathrm{pH}$ de jabones comerciales, tal como lo evidencian resultados obtenidos en una evaluación realizada a 29 jabones de marcas tanto comerciales como de laboratorios médicos [24]. Resultados similares reportaron Alvarado y Alvarado [25], al comparar el pH de 8 marcas de jabones comerciales.

Los resultados obtenidos experimentalmente tanto en la extracción del aceite esencial como en la saponificación, se utilizaron para realizar los diagramas de bloques para cada proceso y sus respectivos balances de masa (figuras 5 y 6 ). En cada caso se indican las corrientes de flujo másico $\left(\mathrm{m}_{\mathrm{i}}\right)$, molares $\left(n_{i}\right)$, las fracciones másicas $\left(X_{i}\right)$ y molares $\left(Y_{i}\right)$.

\section{Conclusión}

En el trabajo realizado se utilizó el aceite esencial de romero para un fin diferente al comúnmente empleado, demostrando que es posible usarlo como extracto en la industria cosmética. EI rendimiento obtenido para la extracción del aceite esencial con el método por arrastre de vapor, se ubicó dentro de los parámetros establecidos en la bibliografía consultada, sin embargo este podría incrementarse añadiendo la operación unitaria de secado antes de la molienda.

El alto rendimiento en la elaboración del jabón se debe a las trazas de glicerina que quedaron en este, razón por la cual es necesario mejorar la operación de separación para este proceso. El pH que se registró para este jabón, se compara con el de los jabones comerciales, así como también por aquellos elaborados por laboratorios médicos.

El procedimiento aquí descrito podría servir de referencia para el sector industrial, cuyo interés sea el desarrollo de nuevos productos basados en componentes de origen natural.

\section{Agradecimientos}

$\mathrm{Se}$ agradece al Instituto Nacional de Investigaciones Agropecuarios (INIAP), Estación Experimental Portoviejo, por facilitar el acceso a sus laboratorios y uso de sus equipos, para realizar parte del desarrollo experimental de la investigación.

\section{Referencias}

[1] M. T. López Luengo, "El romero. Planta aromática con efectos antioxidantes", Offarm Farm. y Soc., vol. 27, n. ${ }^{7}$, pp. 60-63, Jul. 2008.

[2] M. Hyldgaard, T. Mygind, y R. L. Meyer, "Essential oils in food preservation: Mode of action, synergies, and interactions with food matrix components", Front. Microbiol., vol. 3, n. ${ }^{\circ}$ 12, 2012. https://doi:10.3389/fmicb.2012.00012

[3] L. Sánchez-González, M. Vargas, C. González-Martínez, A. Chiralt, y M. Cháfer, "Use of Essential Oils in Bioactive Edible Coatings: A Review", Food Eng. Rev., vol. 3, n 1, pp. 1-16, 2011. https://doi:10.1007/s12393-010-9031-3

[4] R. Ávila, A. Navarro, O. Vera, R. Dávila, N. Melgoza, y R. Meza, "Romero (Rosmarinus officinalis): Una revisión de sus usos no culinarios", Rev. Cienc. y Mar, vol. XV, n. ${ }^{4}$ 43, pp. 23-36, Abr. 2011.

[5] C. A. C. Barrera y G. E. Acosta, "Actividad antibacteriana y determinación de la composición química de los aceites esenciales de romero (Rosmarinus officinalis), tomillo (Thymus vulgaris) y cúrcuma (Curcuma longa) de Colombia", Revista Cubana de Plantas Medicinales, vol. 18, n. 2, pp. 237-246, Jun. 2013.

[6] F. Bakkali, S. Averbeck, D. Averbeck, y M. Idaomar, "Biological effects of essential oils - A review", Food Chem. Toxicol., vol. 46, n² 2, pp. 446-475, 2008. https://doi.org/10.1016/j.fct.2007.09.106 
[7] J. Armijo C., E. Vicuña G., P. Romero y Otiniano, C. Condorhuamán C., y B. Hilario R., "Modelamiento y simulación del proceso de extracción de aceites esenciales mediante la destilación por arrastre con vapor", Rev. Peru. Química e Ing. Química, vol. 15, n. ${ }^{2}$, pp. 1927, 2012.

[8] A. Lamarque, J. Zygadlo, D. Labuckas, L. López, M. Torres, y D. Maestri, Fundamentos teorico-practicos de química orgánica, Argentina: Editorial Brujas, 2008.

[9] M. Rodríguez Álvarez, L. Alcaraz Meléndez, y S. M. Real Cosío, Procedimientos para la extracción de aceites esenciales en plantas aromáticas, México: Centro de Investigaciones Biológicas del Noroeste, S.C., 2012.

[10] J. L. Ríos, "Essential oils: What they are and how the terms are used and defined", Essential Oils in Food Preservation, Flavor and Safety

V. R. Preedy, Ed. Elsevier Inc., 2015, pp. 3-10. https://doi.org/10.1016/B978-0-12-416641-7.00001-8

[11] I. T. Carvalho, B. N. Estevinho, y L. Santos, "Application of microencapsulated essential oils in cosmetic and personal healthcare products - A review", Int. J. Cosmet. Sci., vol. 38, n. ${ }^{2}$ 2, pp. 109-19, 2016. https://doi:10.1111/ics.12232

[12] P. G. Kareru, J. M. Keriko, G. M. Kenii, G. T. Thiong'o, A. N. Gachanja, y H. N. Mukiira, "Antimicrobial activities of skincare preparations from plant extracts", African J. Tradit. Complement. Altern. Med., vol. 7, n³ 3, pp. 214-218, 2010. https://doi:10.4314/aitcam v7i3.54777

[13] I. Regla, E. Vázquez, D. Cuervo, y A. Neri, "La química del jabón y algunas aplicaciones", Revista Digital Universitaria, vol. $15, n^{0} 5$, May. 2014.

[14] R. S. McDaniel, JABONES ESENCIALES. Barcelona: Editorial Paidotribo, 2002

[15] J. A. Neves, J. A. Neves, y R. de C. M. Oliveira, "Pharmacological and biotechnological advances with Rosmarinus officinalis L", Expert Opin. Ther. Pat., vol. 28, n. ${ }^{5}$, pp. 399-413, 2018.

https://doi:10.1080/13543776.2018.1459570

[16] N. Castillo y J. Mendoza. (2012) Manual de prácticas para el laboratorio de química orgánica I, Man. Prácticas Química Orgánica, vol. 1. [Online]. Disponible en:

http://depa.fquim.unam.mx/amyd/archivero/Manual2016-1_31098.pdf
[17] J. Juárez, A. Castro, y J. Jaúregui, "Composición química, actividad antibacteriana del aceite esencial de Citrus sinensis L. (Naranja dulce) y formulación de una forma farmacéutica", Cienc. Invest., vol. 13, n. ${ }^{\circ}$ 1, pp. 9-13, 2010.

[18] C. Guerrero, "Diseño de una planta de fabricación de jabón a partir de aceites vegetales usados", Tesis de grado, Universidad de Almería, Almería, España, Jun. 2014

[19] R. M. Felder y Ronald W. Rousseau, Principios elementales de los procesos químicos, $3^{\mathrm{a}}$ ed. México D.F.: Editorial Limusa, 2004.

[20] A. I. Terry, S. Rodríguez, R. C. Bermúdez, M. Fernández, I. Aguilera, y J. Buzón, "Evaluación de un inóculo para el arranque de reactores UASB empleados en el tratamiento en el tratamiento de residual líquido de café", Rev. Cuba. Química, vol. 17, n. ${ }^{1}$ 1, pp. 206-211, 2005.

[21] A. Angioni et al., "Chemical composition, plant genetic differences, antimicrobial and antifungal activity investigation of the essential oil of Rosmarinus officinalis L.", J. Agric. Food Chem., vol. 52, n. 11 , p. 3530-5, Jun. 2004.

[22] D. M. Bonilla et al., "Efecto del aceite esencial de Rosmarinus officinalis sobre Porphyromonas gingivalis cultivada in vitro", Rev. Colomb. Ciencias Químico-Farmacéuticas, vol. 45, n. ${ }^{2}$ 2, pp. 275-287, 2016. https://doi.org/10.15446/rcciquifa.v45n2.59942

[23] Y. Valverde y J. Leonardo, "Extracción y caracterización del aceite esencial del romero (Rosmarinus Officinalis) por el método de arrastre de vapor obtenida en estado fresco y secado convencional", Tesis de grado, Universidad Nacional del Centro del Perú, Tarma, Perú, 2011.

[24] I. D'Santiago y M. E. Vivas de Marcano, "El pH de los jabones en la industria cosmetica de Mexico", DERMATOLOGIA Venez., vol. 34, n.o 3, pp. 119-120, 1996.

[25] E. E. Alvarado Carranza y M. Y. Alvarado Leiva, "pH de jabones de tocador en barra comercializados en el distrito de Trujillo - Marzo 2012", Universidad Nacional de Trujillo, Trujillo, Perú, May. 2012 